\title{
Evaluación de impacto ambiental de una radio bases celulares instalada desde el nivel del suelo en el Ecuador
}

\author{
Environmental impact assessment of the cellular base stations installed from \\ the ground level in Ecuador \\ Daniela María Llanos Campaña. ${ }^{1}$ \\ Recibido: 12-09-2019 / Revisado: 22-10-2019/Aceptado:13-11-2019/ Publicado: 07-12-2019
}

\begin{abstract}
DOI: https://doi.org/10.33262/cienciadigital.v3i4.2.1022

With the technology development and technological advances in mobile phones, it is common to observe a worldwide implementation of cellular base stations to provide a highquality service to the users of the different mobile phone operators.

Due to this increase and with the interest to guarantee the environmental and social wellbeing, this article evaluates the environmental impacts caused by the construction, operationmaintenance and closure-abandonment of the cell base stations raised from the ground level (tower type) in the country, using the methodology presented by the Ministry of Environment of Ecuador described in its "Study of potential environmental impacts and vulnerability related to chemical substances and treatment of hazardous wastes in the productive sector of Ecuador"

In this sense, 65 interactions have been identified between the activities involved in the installation, operation and closure of the cellular base station with the environmental and social components; of which $65 \%$ correspond to non-significant negative impacts, $11 \%$ insignificant negative and $3 \%$ significant negative, in the same way several positive impacts have been identified representing $11 \%$ of non-significant positive impacts and $3 \%$ for nonsignificant and very significant positive impacts respectively.

Keywords: Environmental assessment, cellular base station, impacts, communication, environment.

\section{Resumen}

Con el desarrollo de las tecnologías de información y avance tecnológico en los modelos de telefonía celular, cada vez es frecuente observar a nivel mundial la implementación de estaciones base celular para brindar un servicio de calidad a los usuarios de las distintas operadoras de telefonía móvil. Debió a este aumento y con el interés de garantizar el bienestar ambiental y social el presente artículo evalúa los impactos ambientales ocasionados por la construcción, operación-mantenimiento y cierre-abandono, de las estaciones base celular levantadas desde el nivel del suelo (tipo torre) en el país, empleando la metodología presentada por el Misterio de Ambiente del Ecuador descrito en el "Estudio de potenciales
\end{abstract}

\footnotetext{
${ }^{1}$ Escuela Superior Politécnica de Chimborazo, Facultad de Ciencias, Orellana, Ecuador, danibella10@hotmail.com
} 
impactos ambientales y vulnerabilidad relacionada con las sustancias químicas y tratamiento de desechos peligrosos en el sector productivo del Ecuador"

En este contexto se han identificado un total de 65 interacciones entre las actividades que conllevan la instalación, funcionamiento y cierre de estas infraestructuras con los componentes ambientales y sociales; de los cuales el $65 \%$ corresponden a impactos negativos no significativos, $11 \%$ negativos poco significativos y $3 \%$ negativos significativos, de la misma manera se han identificados impactos positivos representando de esta manera un $11 \%$ de impactos positivos no significativos y un $3 \%$ para impactos positivos poco y muy significativos respectivamente.

Palabras claves: Evaluación ambiental, estación base celular, impactos, comunicación, medio ambiente.

\section{Introducción}

La industria de la comunicación en los últimos años ha tenido un crecimiento acelerado, en gran parte por el avance tecnológico a nivel mundial. Considerando el informe de la Corporación Interamericana de Inversiones (CII) el acceso a la telefonía móvil se incrementó a más de $70 \%$ en 2017 en América Latina y el Caribe además se señala la importancia del servicio de telecomunicaciones para cumplir la los objetivos de desarrollo sostenible de las Organización de las Naciones Unidas (ONU).

En este contexto Ecuador a través Agencia de Regulación y Control de las Telecomunicaciones hasta el año 2017 concedió la instalación de 2579 radio bases con tecnología 4G en el territorio ecuatoriano, información publicada en el sitio web de dicha entidad. La radio base celular también conocidas como estación base de telefonía móvil o simplemente estación base celular, está definida por la Canadian radio televisión and Telecommunications Commission como una estación conformada por diferentes dispositivos y circuitos eléctricos que se encuentran instaladas en un lugar firme, misma que permiten la comunicación entre los usuarios de telefonía móvil.

Piñeres-Espitia, G. (2010), señala de igual forma que una radio base de telefonía celular está conformada por un sistema de radiofrecuencia (RF), un sistema de microondas (MW), un sistema radiante los cuales están constituidos por equipos tales como antenas y cables los mismos que son instalados en mástiles, un sistema de energía fijo y de reserva (baterías) ubicados en el cuarto de equipos encontrándose además en esta instalación varios equipos de telefonía móvil de diferente tecnología (GSM y 3G). Existen diferentes tipos de estructuras en las que cuales se montan los equipos antes mencionados, debido a que las antenas requieren de un soporte físico de distinta altura para que se lleve a cabo la comunicación inalámbrica Cáceres, V. (2016), pudiendo así levantarse infraestructuras desde el nivel del suelo o sobre techos de edificios de variada altura. En el primer caso se pueden indicar las estructuras tipo torre y monopolo Piñeres-Espitia, G. (2010), siendo este objeto del presente estudio. Las actividades que conllevan a la instalación de estas estructuras pueden variar dependiendo el sitio de instalación ya sea una zona urbana o rural, causando así varios impactos en el entorno. Belmonte, P. y Miralles, P. (2004) expone los impactos sociales, 
ambientales y urbanísticos por la instalación y funcionamiento de las redes de telefonía móvil.

En la actualidad en el Ecuador la Autoridad Ambiental a través del Código Orgánico del Ambiente emitido mediante Registro Oficial Suplemento 983 de 12 abril de 2017 en su última modificación del 21 agosto de 2018, establece en su artículo 174, del Capítulo III referente a la regularización ambiental, que se elaborará y actualizará el catálogo de actividades, de los proyectos, obras o actividades existentes en el país que deban regularizarse, en función de la magnitud del impacto o riesgo ambiental que puedan generar.

En este sentido, en base a la información obtenida del Sistema único de información ambiental del Ministerio de Ambiente (http://suia.ambiente.gob.ec/catalogo_ambiental) la construcción y/u operación de radio bases celulares corresponde a la obtención de un registro ambiental. Es por ello, que con la finalidad de determinar el grado de afectación que produce esta actividad se ha empleado la metodología presentada por esta entidad en su "Estudio de potenciales impactos ambientales y vulnerabilidad relacionada con las sustancias químicas y tratamiento de desechos peligrosos en el sector productivo del Ecuador", considerando de este modo las actividades de construcción, funcionamiento y cierre de una radio base celular instalada desde el suelo tipo torre.

\section{Metodología}

Como ya se ha mencionado anteriormente, la siguiente metodología fue tomada del estudio efectuado por el Ministerio de Ambiente.

\section{Identificación de impactos}

Para la identificación de impactos se considera una matriz de interacción entre los factores ambientales (Tabla 1) y las actividades involucras por el proyecto.

Tabla 1. Componentes y factores ambientales para identificación de impactos ambientales.

\begin{tabular}{|l|l|}
\hline Componentes & Factores \\
\hline \multirow{2}{*}{ Recurso aire } & Calidad de aire (gases de combustión, MP, olores) \\
\cline { 2 - 2 } & Nivel de ruido y vibraciones \\
\hline Recurso agua & Calidad de agua (generación de efluentes) \\
\hline Recurso suelo & Calidad del suelo \\
\hline Desechos & Generación de desechos sólidos \\
\hline \multirow{4}{*}{ Proceso geomorfodinámicos } & Erosión \\
\cline { 2 - 2 } & Geomorfología \\
\cline { 2 - 2 } & Inestabilidad \\
\hline \multirow{4}{*}{ Sedio biótico } & Flora \\
\hline & Fauna \\
\cline { 2 - 2 } & Ecosistemas \\
\hline \multirow{5}{*}{ Socioeconómico } & Actividades comerciales \\
\cline { 2 - 2 } & Empleo \\
\cline { 2 - 2 } & Aspectos paisajísticos \\
\cline { 2 - 2 } & Riesgos a la población \\
\cline { 2 - 2 } & Servicios básicos \\
\cline { 2 - 2 } & Calidad de vida de las comunidades \\
\cline { 2 - 2 } & Salud ocupacional y seguridad laboral \\
\hline
\end{tabular}

Fuente: Ministerio de Ambiente. 


\section{Evaluación de impactos}

La valoración de los impactos ambientales está basada en dos criterios; el primero, un análisis cuantitativo en el cual se obtiene la magnitud del impacto y el segundo un análisis cualitativo donde se asigna la importancia del impacto, utilizando en ambos casos una escala numérica. Magnitud del impacto

La magnitud del impacto considera seis variables, mismas que se encuentran expresadas en ecuación 1 y detalladas en la tabla 2:

$$
\begin{gathered}
M=\text { Carácter } \times \text { Probabilidad } \times(\text { Persistencia }+ \text { Reversibilidad }+ \text { Intensidad }+ \\
\text { Extensión }) \text { Ecuación }(1)
\end{gathered}
$$

\begin{tabular}{|c|c|c|}
\hline Parámetro & Escala & Definición \\
\hline \multirow{2}{*}{$\begin{array}{l}\text { Carácter } \\
\text { ( C ) }\end{array}$} & Benéfico (1) & Impacto es positivo \\
\hline & Detrimente $(-1)$ & Impacto es negativo o adverso \\
\hline \multirow{3}{*}{$\begin{array}{l}\text { Intensidad } \\
\text { (I) }\end{array}$} & Baja (1) & Si el efecto es sutil o casi imperceptible \\
\hline & Media (2) & Si el efecto es notable pero difícil de medirse o de monitorear. \\
\hline & Alta (3) & Si el efecto es obvio o notable. \\
\hline \multirow{3}{*}{$\begin{array}{c}\text { Extensión } \\
\text { (E) }\end{array}$} & Puntual (1) & Si el efecto está limitado a la "huella" del impacto \\
\hline & Local (2) & Si el efecto se concentra en los límites de área de influencia del proyecto \\
\hline & Regional (3) & Si el efecto o impacto sale de los límites del área del proyecto. \\
\hline \multirow[t]{2}{*}{ Reversibilidad } & A corto plazo (1) & $\begin{array}{l}\text { Cuando un impacto puede ser asimilado por el propio entorno en el } \\
\text { tiempo. }\end{array}$ \\
\hline & A largo plazo (2) & $\begin{array}{l}\text { Cuando el efecto no es asimilado por el entorno o si es asimilado toma } \\
\text { un tiempo considerable }\end{array}$ \\
\hline \multirow{3}{*}{$\begin{array}{l}\text { Probabilidad } \\
\text { (Pr) }\end{array}$} & Poco Probable $(0.1)$ & El impacto tiene una baja probabilidad de ocurrencia \\
\hline & Probable $(0.5)$ & El impacto tiene una media probabilidad de ocurrencia \\
\hline & Cierto (1) & El impacto tiene una alta probabilidad de ocurrencia \\
\hline \multirow{2}{*}{$\begin{array}{l}\text { Persistencia } \\
\text { (P) }\end{array}$} & Temporal (1) & El tiempo requerido para la fase de construcción. \\
\hline & Permanente (2) & El tiempo requerido para la fase de operación \\
\hline
\end{tabular}

Tabla 2. Definición y valoración de la magnitud del impacto

Fuente: Ministerio de Ambiente.

\section{Importancia del impacto}

La valoración de la importancia es un valor intrínseco dependiendo la experticia del investigador o grupo de investigadores, por lo cual para el presente artículo se considerará una ponderación de un rango de 1 a 10 como se detalla a continuación.

Tabla 3. Valoración de la importancia del impacto.

\begin{tabular}{|c|l|}
\hline Escala & \multicolumn{1}{c|}{ Importancia } \\
\hline $1-3$ & Poco importante \\
\hline $4-6$ & Medianamente importante \\
\hline $7-9$ & Importante \\
\hline 10 & Muy importante \\
\hline
\end{tabular}

Fuente: Elaboración propia 


\section{Jerarquización de los impactos}

El producto de la magnitud y la importancia da como resultado el valor total del impacto total, el mismo que es ponderado dentro del rango de valores y asignado el nivel de significancia como se muestra en la tabla 4.

$$
\text { Valor total del impacto }=M \times I \text { Ecuación (2) }
$$

Tabla 4. Rango porcentual y nivel de significancia de los impactos

\begin{tabular}{|c|l|}
\hline Rango & Significancia \\
\hline $81-100$ & Muy significativo positivo \\
\hline $61-80$ & Significativo positivo \\
\hline $41-60$ & Medianamente significativo positivo \\
\hline $21-40$ & Poco significativo positivo \\
\hline $0-20$ & No significativo positivo \\
\hline$(-) 1-20$ & No significativo negativo \\
\hline$(-) 21-40$ & Poco significativo negativo \\
\hline$(-) 41-60$ & Medianamente significativo negativo \\
\hline$(-) 61-80$ & Significativo negativo \\
\hline$(-) 81-100$ & Muy significativo negativo \\
\hline
\end{tabular}

Fuente: Ministerio de Ambiente

\section{Resultados}

En base a la metodología antes descrita, se identificaron nueve actividades principales, las mismas que fueron clasificadas de acuerdo a las fases del proyecto y se muestran a continuación:

Fase de construcción:

- Apertura de acceso al sitio de instalación.

- Remoción de cobertura vegetal.

- Adecuación del terreno (excavación y/o compactación).

- Construcción de cerramiento.

- Instalación de estructura y equipos.

\section{Fase de Operación y mantenimiento:}

- Funcionamiento de radio base celulares.

- Mantenimiento de estructura y equipos.

\section{Fase de cierre:}

- Desmantelamiento de estructura y equipos.

- Limpieza general del área.

Se analizaron de este modo los componentes ambientales afectados por estas actividades, obteniendo así un total de 65 interacciones.

Posteriormente, estos impactos fueron evaluados y clasificados de acuerdo a las fases de construcción, operación- mantenimiento y cierre- abandono, reconociendo de este modo en la fase inicial del proyecto (tabla 5) 34 impactos no significativos negativos, 6 impactos poco significativos negativos, 4 impactos no significativos positivos y 1 impacto poco significativo positivo. En esta fase los impactos negativos más relevantes aunque con nivel bajo están relacionados con la alteración al paisaje natural y la afectación a la flora y fauna, seguidos en 
menor proporción la contaminación al aire por la emisión de partículas y ruido, la generación de desechos a variada escala, la alteración que puede suscitarse en el recurso suelo por la apertura y modificaciones del área de instalación, sumándose igualmente los riesgos a los trabajadores, por lo contrario, el impacto positivo identificado en esta fase es la generación de empleo. Según Martin, M. (2001) indica que se presenta una escasa magnitud de los impactos ambientales que pudiesen ocasionarse al relieve, geología y al suelo por los movimientos de tierras, nivelación y compactación y cimentación de la torre, además incluye los impactos al recurso agua, vegetación o la fauna durante la implementación. Sin embargo, la magnitud de los impactos se intensifica sobre el entorno paisajístico y la población en determinadas ocasiones.

Además de estos impactos Belmonte, P. y Miralles, P. (2004) señalan los posibles daños al medio natural, suelo y avifauna por la instalación de las estaciones base celular.

Por su parte, en la tabla 6 se muestran los impactos durante la fase de operaciónmantenimiento donde se detectan: 2 impactos muy significativos positivos los cuales están relacionados con la mejora de la calidad de vida de las comunidades por la entre ga del servicio básico como es el caso de la comunicación, 1 impacto no significativo positivo vinculado con la generación de empleo por el mantenimiento de la estación base celular, 3 impactos no significativo negativo como son el riesgo a la población, a la fauna y flora del área circundante debido a la emisión de ondas electromagnéticas (radiaciones no ionizantes), debido a que diversos estudios indican posibles afecciones como menciona: Hyland, G. (2000) la existe evidencia de que la radiación pulsada de baja intensidad que se usa en las radio base celular puede ejercer influencias sutiles no térmicas, por su parte Monsalve, $\mathrm{O} \&$ Mosquera, J (2018) en su investigación también indican que la contaminación ambiental por la radiación no ionizante tienden a variar en función del área de estudio, presencia de edificaciones y de la distancia en la que se encuentras las estaciones base celular.

También se reconocen en esta fase 3 impactos poco significativo negativo concernientes al impacto a los aspectos paisajísticos producido por la presencia de la infraestructura como cita Belmonte, P. y Miralles, P. (2004) incluyendo además la contaminación electromagnética ya antes citada, de la misma manera durante el mantenimiento se reconoce la generación de desechos sólidos como es el cambio de baterías ya que estas son a base de plomo y al ser un elemento bioacumulable puede ocasionar afectaciones al ambiente y a la salud humana por lo cual deben tener una adecuada disposición final Ornetta, V (2017) y los impactos a la salud ocupacional y seguridad laboral durante los mantenimientos.

En la tabla 7, concerniente a los impactos en la fase de cierre y abandono se identifican que debido al desmantelamiento de la infraestructura se provocan dos impactos significativos negativos, esto ocasionado por la pérdida del servicio de comunicación y por ende afectando de forma negativa el estilo de calidad de vida en la ciudadanía, por esta misma actividad se produce la generación de desechos siendo un impacto poco significativo negativo, y dos impactos no significativos negativos asociados al nivel de ruido, vibraciones y la salud ocupacional y seguridad laboral, de la misma manera estos impactos son ocasionados por la 
limpieza del área. Sin embargo, se distinguen impactos positivos como son la generación de empleo, y el retorno al paisaje natural eliminando así el impacto visual.

Finalmente se puede resaltar que usando esta metodología de manera global un $65 \%$ corresponden a impactos negativos no significativos, $11 \%$ negativos poco significativos y $3 \%$ negativos significativos, por su parte también los impactos positivos se ven representando por un $11 \%$ de impactos positivos no significativos y un $3 \%$ para impactos positivos poco y muy significativos respectivamente.

Por su parte Silva, C (2017) en su tesis señala que más del 50\% de estaciones base celular a nivel piso presentan un impacto medio, en cuya evaluación incluye el tamaño de la muestra.

Tabla 5. Evaluación de Impactos ambientales en la fase de construcción.

\begin{tabular}{|c|c|c|c|c|c|c|c|c|c|c|c|}
\hline & & & \multicolumn{5}{|c|}{ CONSTRUCCIÓN } & \multicolumn{2}{|c|}{$\begin{array}{c}\text { OPERACIÓN Y } \\
\text { MANTENIMIENTO }\end{array}$} & $\begin{array}{l}\text { CIERRE Y } \\
\text { ABANDONO }\end{array}$ & \\
\hline Componctestes & Factores & 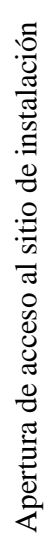 & 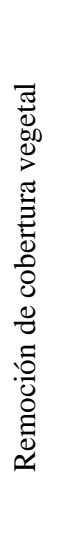 & 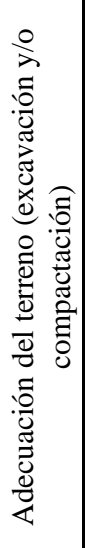 & 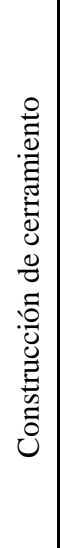 & 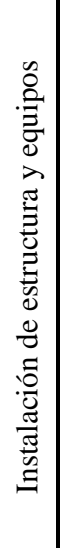 & 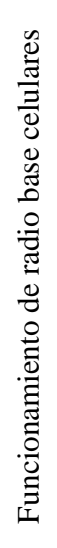 & 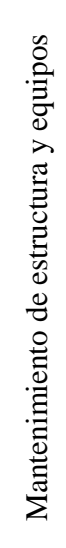 & 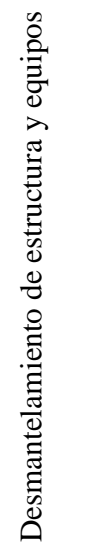 & 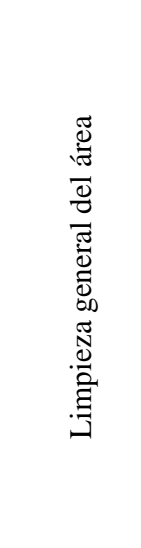 & 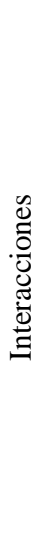 \\
\hline \multirow{2}{*}{ Recurso aire } & Calidad de aire (gases de combustión, MP, olores) & $\mathrm{X}$ & $\mathrm{X}$ & $\mathrm{X}$ & & & & & & $\mathrm{X}$ & 4 \\
\hline & Nivel de ruido y vibraciones & $\mathrm{X}$ & $\mathrm{X}$ & $\mathrm{X}$ & $\mathrm{X}$ & $\mathrm{X}$ & & & $\mathrm{X}$ & & 6 \\
\hline Recurso agua & Calidad de agua (generación de efluentes) & & & & & & & & & & 0 \\
\hline Recurso suelo & Calidad del suelo & & & & & & & & & & 0 \\
\hline Desechos & Generación de desechos sólidos & $\mathrm{X}$ & $\mathrm{X}$ & $\mathrm{X}$ & $\mathrm{X}$ & $\mathrm{X}$ & & $\mathrm{X}$ & $\mathrm{X}$ & $\mathrm{X}$ & 8 \\
\hline \multirow{3}{*}{$\begin{array}{l}\text { Proceso } \\
\text { geomorfodinámicos }\end{array}$} & Erosión & $\mathrm{X}$ & $\mathrm{X}$ & $\mathrm{X}$ & & & & & & & 3 \\
\hline & Geomorfología & $\mathrm{X}$ & $\mathrm{X}$ & $\mathrm{X}$ & & & & & & & 3 \\
\hline & Inestabilidad & & $\mathrm{X}$ & $\mathrm{X}$ & & & & & & & 2 \\
\hline \multirow{3}{*}{ Medio biótico } & Flora & $\mathrm{X}$ & $\mathrm{X}$ & $\mathrm{X}$ & & & $\mathrm{X}$ & & & & 4 \\
\hline & Fauna & $\mathrm{X}$ & $\mathrm{X}$ & $\mathrm{X}$ & & & $\mathrm{X}$ & & & & 4 \\
\hline & Ecosistemas & $\mathrm{X}$ & $\mathrm{X}$ & $\mathrm{X}$ & & & & & & & 3 \\
\hline \multirow{7}{*}{ Socio económico } & Actividades comerciales & & & & & & & & & & 0 \\
\hline & Empleo & $\mathrm{X}$ & $\mathrm{X}$ & $\mathrm{X}$ & $\mathrm{X}$ & $\mathrm{X}$ & & $\mathrm{X}$ & $\mathrm{X}$ & $\mathrm{X}$ & 8 \\
\hline & Aspectos paisajísticos & $\mathrm{X}$ & $\mathrm{X}$ & $\mathrm{X}$ & $\mathrm{X}$ & $\mathrm{X}$ & $\mathrm{X}$ & & $\mathrm{X}$ & & 7 \\
\hline & Riesgos a la población & & & & & & $\mathrm{X}$ & & & & 1 \\
\hline & Servicios básicos & & & & & & $\mathrm{X}$ & & $\mathrm{X}$ & & 2 \\
\hline & Calidad de vida de las comunidades & & & & & & $\mathrm{X}$ & & $\mathrm{X}$ & & 2 \\
\hline & Salud ocupacional y seguridad laboral & $\mathrm{X}$ & $\mathrm{X}$ & $\mathrm{X}$ & $\mathrm{X}$ & $X$ & & $\mathrm{X}$ & $X$ & $\mathrm{X}$ & 8 \\
\hline
\end{tabular}

Fuente: Elaboración propia 
Tabla 6. Evaluación de Impactos ambientales en la fase de operación-mantenimiento.

\begin{tabular}{|c|c|c|c|c|c|c|c|c|c|c|c|}
\hline Actividad & Factores & نَّ & 胥 & : & 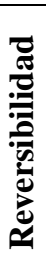 & 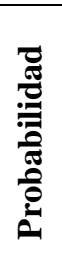 & 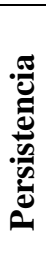 & 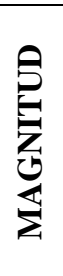 & 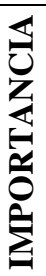 & $\begin{array}{l}\text { IMPACTO } \\
\text { TOTAL }\end{array}$ & $\begin{array}{l}\text { SIGNIFICANCIA } \\
\text { DEL IMPACTO }\end{array}$ \\
\hline \multirow{11}{*}{$\begin{array}{c}\text { Apertura de } \\
\text { acceso al sitio } \\
\text { de instalación }\end{array}$} & $\begin{array}{l}\text { Calidad de aire } \\
\text { (gases de } \\
\text { combustión, } \\
\text { MP, olores) }\end{array}$ & -1 & 2 & 2 & 1 & 1 & 1 & -6 & 4 & -24 & $\begin{array}{c}\text { POCO } \\
\text { SIGNIFICATIVO } \\
\text { NEGATIVO }\end{array}$ \\
\hline & $\begin{array}{l}\text { Nivel de ruido } \\
\text { y vibraciones }\end{array}$ & -1 & 2 & 2 & 1 & 1 & 1 & -6 & 4 & -24 & $\begin{array}{c}\text { POCO } \\
\text { SIGNIFICATIVO } \\
\text { NEGATIVO }\end{array}$ \\
\hline & $\begin{array}{l}\text { Generación de } \\
\text { desechos } \\
\text { sólidos }\end{array}$ & -1 & 3 & 1 & 1 & 1 & 1 & -6 & 3 & -18 & $\begin{array}{c}\text { NO SIGNIFICATIVO } \\
\text { NEGATIVO }\end{array}$ \\
\hline & Erosión & -1 & 2 & 1 & 1 & 0,5 & 1 & 2,5 & 5 & $-12,5$ & $\begin{array}{c}\text { NO SIGNIFICATIVO } \\
\text { NEGATIVO }\end{array}$ \\
\hline & Geomorfología & -1 & 2 & 1 & 1 & 0,5 & 1 & 2,5 & 4 & -10 & $\begin{array}{c}\text { NO SIGNIFICATIVO } \\
\text { NEGATIVO }\end{array}$ \\
\hline & Flora & -1 & 2 & 1 & 1 & 2 & 1 & $\begin{array}{c}- \\
10\end{array}$ & 4 & -40 & $\begin{array}{c}\text { POCO } \\
\text { SIGNIFICATIVO } \\
\text { NEGATIVO }\end{array}$ \\
\hline & Fauna & -1 & 2 & 1 & 1 & 2 & 1 & $\begin{array}{c}- \\
10 \\
\end{array}$ & 4 & -40 & $\begin{array}{c}\text { POCO } \\
\text { SIGNIFICATIVO } \\
\text { NEGATIVO } \\
\end{array}$ \\
\hline & Ecosistemas & -1 & 2 & 2 & 1 & 0,5 & 1 & -3 & 3 & -9 & $\begin{array}{c}\text { NO SIGNIFICATIVO } \\
\text { NEGATIVO }\end{array}$ \\
\hline & Empleo & 1 & 2 & 1 & 1 & 1 & 1 & 5 & 7 & 35 & $\begin{array}{c}\text { POCO } \\
\text { SIGNIFICATIVO } \\
\text { POSITIVO } \\
\end{array}$ \\
\hline & $\begin{array}{l}\text { Aspectos } \\
\text { paisajísticos }\end{array}$ & -1 & 3 & 2 & 1 & 1 & 1 & -7 & 5 & -35 & $\begin{array}{c}\text { POCO } \\
\text { SIGNIFICATIVO } \\
\text { NEGATIVO } \\
\end{array}$ \\
\hline & $\begin{array}{l}\text { Salud } \\
\text { ocupacional y } \\
\text { seguridad } \\
\text { laboral } \\
\end{array}$ & -1 & 2 & 1 & 1 & 0,5 & 1 & 2,5 & 8 & -20 & $\begin{array}{c}\text { NO SIGNIFICATIVO } \\
\text { NEGATIVO }\end{array}$ \\
\hline \multirow{7}{*}{$\begin{array}{l}\text { Remoción de } \\
\text { cobertura } \\
\text { vegetal del } \\
\text { sitio de } \\
\text { instalación }\end{array}$} & $\begin{array}{l}\text { Calidad de aire } \\
\text { (gases de } \\
\text { combustión, } \\
\text { MP, olores) } \\
\end{array}$ & -1 & 2 & 2 & 1 & 0,5 & 1 & -3 & 3 & -9 & $\begin{array}{c}\text { NO SIGNIFICATIVO } \\
\text { NEGATIVO }\end{array}$ \\
\hline & $\begin{array}{l}\text { Nivel de ruido } \\
\text { y vibraciones }\end{array}$ & -1 & 2 & 2 & 1 & 0,5 & 1 & -3 & 3 & -9 & $\begin{array}{l}\text { NO SIGNIFICATIVO } \\
\text { NEGATIVO }\end{array}$ \\
\hline & $\begin{array}{l}\text { Generación de } \\
\text { desechos } \\
\text { sólidos }\end{array}$ & -1 & 2 & 1 & 1 & 0,5 & 1 & 2,5 & 3 & $-7,5$ & $\begin{array}{l}\text { NO SIGNIFICATIVO } \\
\text { NEGATIVO }\end{array}$ \\
\hline & Erosión & -1 & 2 & 1 & 1 & 0,5 & 1 & 2,5 & 4 & -10 & $\begin{array}{c}\text { NO SIGNIFICATIVO } \\
\text { NEGATIVO }\end{array}$ \\
\hline & Geomorfología & -1 & 2 & 1 & 1 & 0,5 & 1 & 2,5 & 3 & $-7,5$ & $\begin{array}{c}\text { NO SIGNIFICATIVO } \\
\text { NEGATIVO }\end{array}$ \\
\hline & Inestabilidad & -1 & 2 & 1 & 1 & 0,5 & 1 & 2,5 & 4 & -10 & $\begin{array}{l}\text { NO SIGNIFICATIVO } \\
\text { NEGATIVO } \\
\end{array}$ \\
\hline & Flora & -1 & 3 & 1 & 1 & 0,5 & 1 & -3 & 4 & -12 & $\begin{array}{l}\text { NO SIGNIFICATIVO } \\
\text { NEGATIVO }\end{array}$ \\
\hline
\end{tabular}


ISSN: 2602-8085

Vol. 3, N 4.2 , p. 179-190, diciembre 2019

\begin{tabular}{|c|c|c|c|c|c|c|c|c|c|c|c|}
\hline & Fauna & -1 & 2 & 1 & 1 & 0,5 & 1 & 2,5 & 3 & $-7,5$ & $\begin{array}{c}\text { NO SIGNIFICATIVO } \\
\text { NEGATIVO }\end{array}$ \\
\hline & Ecosistemas & -1 & 2 & 1 & 1 & 0,5 & 1 & 2,5 & 3 & $-7,5$ & $\begin{array}{c}\text { NO SIGNIFICATIVO } \\
\text { NEGATIVO }\end{array}$ \\
\hline & Empleo & 1 & 1 & 1 & 1 & 0,5 & 1 & 2 & 7 & 14 & $\begin{array}{l}\text { NO SIGNIFICATIVO } \\
\text { POSITIVO }\end{array}$ \\
\hline & $\begin{array}{l}\text { Aspectos } \\
\text { paisajísticos }\end{array}$ & -1 & 3 & 1 & 1 & 0,5 & 1 & -3 & 4 & -12 & $\begin{array}{l}\text { NO SIGNIFICATIVO } \\
\text { NEGATIVO }\end{array}$ \\
\hline & $\begin{array}{l}\text { Salud } \\
\text { ocupacional y } \\
\text { seguridad } \\
\text { laboral }\end{array}$ & -1 & 2 & 1 & 1 & 0,5 & 1 & 2,5 & 8 & -20 & $\begin{array}{l}\text { NO SIGNIFICATIVO } \\
\text { NEGATIVO }\end{array}$ \\
\hline \multirow{12}{*}{$\begin{array}{c}\text { Adecuación } \\
\text { del terreno } \\
\text { (excavación } \\
\text { y/o } \\
\text { compactación) }\end{array}$} & $\begin{array}{l}\text { Calidad de aire } \\
\text { (gases de } \\
\text { combustión, } \\
\text { MP, olores) }\end{array}$ & -1 & 1 & 1 & 1 & 0,1 & 1 & 0,4 & 3 & $-1,2$ & $\begin{array}{l}\text { NO SIGNIFICATIVO } \\
\text { NEGATIVO }\end{array}$ \\
\hline & $\begin{array}{l}\text { Nivel de ruido } \\
\text { y vibraciones }\end{array}$ & -1 & 2 & 1 & 1 & 0,1 & 1 & - & 3 & $-1,5$ & $\begin{array}{l}\text { NO SIGNIFICATIVO } \\
\text { NEGATIVO }\end{array}$ \\
\hline & \begin{tabular}{|l|} 
Generación de \\
desechos \\
sólidos \\
\end{tabular} & -1 & 2 & 1 & 1 & 0,1 & 1 & $0,-$ & 3 & $-1,5$ & $\begin{array}{l}\text { NO SIGNIFICATIVO } \\
\text { NEGATIVO }\end{array}$ \\
\hline & Erosión & -1 & 2 & 1 & 1 & 0,1 & 1 & $\overline{-}$ & 3 & $-1,5$ & $\begin{array}{c}\text { NO SIGNIFICATIVO } \\
\text { NEGATIVO }\end{array}$ \\
\hline & Geomorfología & -1 & 2 & 1 & 1 & 0,1 & 1 & 0,5 & 3 & $-1,5$ & $\begin{array}{c}\text { NO SIGNIFICATIVO } \\
\text { NEGATIVO }\end{array}$ \\
\hline & Inestabilidad & -1 & 1 & 1 & 1 & 0,1 & 1 & - & 3 & $-1,2$ & $\begin{array}{c}\text { NO SIGNIFICATIVO } \\
\text { NEGATIVO }\end{array}$ \\
\hline & Flora & -1 & 1 & 1 & 1 & 0,1 & 1 & 0,4 & 3 & $-1,2$ & $\begin{array}{c}\text { NO SIGNIFICATIVO } \\
\text { NEGATIVO }\end{array}$ \\
\hline & Fauna & -1 & 1 & 1 & 1 & 0,1 & 1 & 0,4 & 3 & $-1,2$ & $\begin{array}{c}\text { NO SIGNIFICATIVO } \\
\text { NEGATIVO }\end{array}$ \\
\hline & Ecosistemas & -1 & 1 & 1 & 1 & 0,1 & 1 & 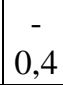 & 3 & $-1,2$ & $\begin{array}{c}\text { NO SIGNIFICATIVO } \\
\text { NEGATIVO }\end{array}$ \\
\hline & Empleo & 1 & 1 & 1 & 1 & 0,5 & 1 & 2 & 7 & 14 & $\begin{array}{l}\text { NO SIGNIFICATIVO } \\
\text { POSITIVO }\end{array}$ \\
\hline & \begin{tabular}{|l}
$\begin{array}{l}\text { Aspectos } \\
\text { paisajísticos }\end{array}$ \\
\end{tabular} & -1 & 1 & 1 & 1 & 0,1 & 1 & 0,4 & 3 & $-1,2$ & $\begin{array}{c}\text { NO SIGNIFICATIVO } \\
\text { NEGATIVO }\end{array}$ \\
\hline & \begin{tabular}{|l|} 
Salud \\
ocupacional y \\
seguridad \\
laboral \\
\end{tabular} & -1 & 2 & 1 & 1 & 0,5 & 1 & 2,5 & 8 & -20 & $\begin{array}{l}\text { NO SIGNIFICATIVO } \\
\text { NEGATIVO }\end{array}$ \\
\hline \multirow{5}{*}{$\begin{array}{c}\text { Construcción } \\
\text { de } \\
\text { cerramiento }\end{array}$} & $\begin{array}{l}\text { Nivel de ruido } \\
\text { y vibraciones }\end{array}$ & -1 & 1 & 1 & 1 & 0,5 & 1 & -2 & 2 & -4 & $\begin{array}{c}\text { NO SIGNIFICATIVO } \\
\text { NEGATIVO }\end{array}$ \\
\hline & \begin{tabular}{|l} 
Generación de \\
desechos \\
sólidos
\end{tabular} & -1 & 2 & 1 & 1 & 0,5 & 1 & 2,5 & 3 & $-7,5$ & $\begin{array}{l}\text { NO SIGNIFICATIVO } \\
\text { NEGATIVO }\end{array}$ \\
\hline & Empleo & 1 & 1 & 1 & 1 & 0,5 & 1 & 2 & 7 & 14 & $\begin{array}{c}\text { NO SIGNIFICATIVO } \\
\text { POSITIVO }\end{array}$ \\
\hline & \begin{tabular}{|l} 
Aspectos \\
paisajísticos
\end{tabular} & -1 & 2 & 1 & 1 & 0,5 & 1 & 2,5 & 5 & $-12,5$ & $\begin{array}{c}\text { NO SIGNIFICATIVO } \\
\text { NEGATIVO }\end{array}$ \\
\hline & $\begin{array}{l}\text { Salud } \\
\text { ocupacional y }\end{array}$ & -1 & 2 & 1 & 1 & 0,5 & 1 & 2,5 & 8 & -20 & $\begin{array}{c}\text { NO SIGNIFICATIVO } \\
\text { NEGATIVO }\end{array}$ \\
\hline
\end{tabular}


ISSN: 2602-8085

\begin{tabular}{|c|c|c|c|c|c|c|c|c|c|c|c|}
\hline & $\begin{array}{l}\text { seguridad } \\
\text { laboral }\end{array}$ & & & & & & & & & & \\
\hline \multirow{5}{*}{$\begin{array}{c}\text { Instalación de } \\
\text { estructura y } \\
\text { equipos }\end{array}$} & $\begin{array}{l}\text { Nivel de ruido } \\
\text { y vibraciones }\end{array}$ & -1 & 2 & 1 & 1 & 0,5 & 1 & 2,5 & 6 & -15 & $\begin{array}{l}\text { NO SIGNIFICATIVO } \\
\text { NEGATIVO }\end{array}$ \\
\hline & $\begin{array}{l}\text { Generación de } \\
\text { desechos } \\
\text { sólidos }\end{array}$ & -1 & 1 & 1 & 1 & 0,5 & 1 & -2 & 7 & -14 & $\begin{array}{c}\text { NO SIGNIFICATIVO } \\
\text { NEGATIVO }\end{array}$ \\
\hline & Empleo & 1 & 1 & 1 & 1 & 0,5 & 1 & 2 & 7 & 14 & $\begin{array}{c}\text { NO SIGNIFICATIVO } \\
\text { POSITIVO }\end{array}$ \\
\hline & \begin{tabular}{|l|} 
Aspectos \\
paisajísticos
\end{tabular} & -1 & 2 & 1 & 1 & 0,5 & 1 & 2,5 & 9 & $-22,5$ & $\begin{array}{c}\text { POCO } \\
\text { SIGNIFICATIVO } \\
\text { NEGATIVO } \\
\end{array}$ \\
\hline & $\begin{array}{l}\text { Salud } \\
\text { ocupacional y } \\
\text { seguridad } \\
\text { laboral }\end{array}$ & -1 & 2 & 1 & 1 & 0,5 & 1 & 2,5 & 8 & -20 & $\begin{array}{c}\text { NO SIGNIFICATIVO } \\
\text { NEGATIVO }\end{array}$ \\
\hline
\end{tabular}

Fuente: Elaboración propia

Tabla 7. Evaluación de impactos ambientales en la fase cierre abandono

\begin{tabular}{|c|c|c|c|c|c|c|c|c|c|c|c|}
\hline Actividad & Factores & Uై & 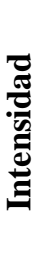 & :0ี & 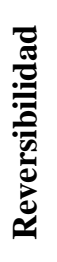 & 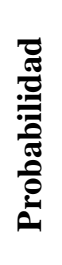 & 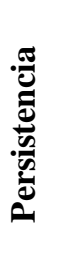 & 总 & 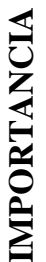 & $\begin{array}{l}\text { IMPACTO } \\
\text { TOTAL }\end{array}$ & $\begin{array}{l}\text { SIGNIFICANCIA } \\
\text { DEL IMPACTO }\end{array}$ \\
\hline \multirow{6}{*}{$\begin{array}{c}\text { Funcionamiento } \\
\text { de radio base } \\
\text { celulares }\end{array}$} & Flora & -1 & 1 & 2 & 1 & 0,5 & 2 & -3 & 5 & -15 & $\begin{array}{l}\text { NO SIGNIFICATIVO } \\
\text { NEGATIVO }\end{array}$ \\
\hline & Fauna & -1 & 1 & 2 & 1 & 0,5 & 2 & -3 & 5 & -15 & $\begin{array}{l}\text { NO SIGNIFICATIVO } \\
\text { NEGATIVO }\end{array}$ \\
\hline & $\begin{array}{l}\text { Aspectos } \\
\text { paisajísticos }\end{array}$ & -1 & 3 & 2 & 1 & 0,5 & 2 & -4 & 9 & -36 & $\begin{array}{c}\text { POCO } \\
\text { SIGNIFICATIVO } \\
\text { NEGATIVO } \\
\end{array}$ \\
\hline & $\begin{array}{l}\text { Riesgos a la } \\
\text { población }\end{array}$ & -1 & 2 & 2 & 1 & 0,5 & 2 & 3,5 & 5 & $-17,5$ & $\begin{array}{c}\text { NO SIGNIFICATIVO } \\
\text { NEGATIVO }\end{array}$ \\
\hline & $\begin{array}{l}\text { Servicios } \\
\text { básicos }\end{array}$ & 1 & 3 & 3 & 1 & 1 & 2 & 9 & 9 & 81 & $\begin{array}{c}\text { MUY } \\
\text { SIGNIFICATIVO } \\
\text { POSITIVO } \\
\end{array}$ \\
\hline & $\begin{array}{l}\text { Calidad de } \\
\text { vida de las } \\
\text { comunidades } \\
\end{array}$ & 1 & 3 & 3 & 1 & 1 & 2 & 9 & 9 & 81 & $\begin{array}{c}\text { MUY } \\
\text { SIGNIFICATIVO } \\
\text { POSITIVO }\end{array}$ \\
\hline \multirow{3}{*}{$\begin{array}{c}\text { Mantenimiento } \\
\text { de estructura y } \\
\text { equipos }\end{array}$} & $\begin{array}{l}\text { Generación } \\
\text { de desechos } \\
\text { sólidos }\end{array}$ & -1 & 2 & 2 & 1 & 0,5 & 2 & 3,5 & 6 & -21 & $\begin{array}{c}\text { POCO } \\
\text { SIGNIFICATIVO } \\
\text { NEGATIVO }\end{array}$ \\
\hline & Empleo & 1 & 1 & 1 & 1 & 0,5 & 2 & 2,5 & 7 & 17,5 & $\begin{array}{l}\text { NO SIGNIFICATIVO } \\
\text { POSITIVO }\end{array}$ \\
\hline & $\begin{array}{l}\text { Salud } \\
\text { ocupacional } \\
\text { y seguridad } \\
\text { laboral }\end{array}$ & -1 & 2 & 1 & 1 & 0,5 & 2 & -3 & 9 & -27 & $\begin{array}{c}\text { POCO } \\
\text { SIGNIFICATIVO } \\
\text { NEGATIVO }\end{array}$ \\
\hline
\end{tabular}

Fuente: Elaboración propia 


\section{Conclusiones}

- Las estaciones base celular en América Latina y el Caribe han aumentado en los últimos años, generando impactos positivos y negativos tanto al medio ambiente como a la sociedad.

- Considerando una estación base celular tipo torre, es decir levantada desde el nivel del suelo se identifica un $83 \%$ de impactos negativos y un $17 \%$ de impactos positivos.

- Aunque la mayoría de los impactos negativos son de baja significancia se reconoce que la generación de desechos, la percepción del paisaje, cambio del uso del suelo y la salud de los trabajadores involucrados durante la ejecución del proyecto son uno de los componentes más afectados.

- Por su parte, dentro de los impactos benéficos se destaca el servicio de telefonía móvil y por consecuente una mejor calidad de vida.

\section{Referencias bibliográficas:}

Barrera-Monsalve, Ó., \& Mosquera-Téllez, J. (2018). Contaminación ambiental por ondas electromagnéticas no ionizantes producto de tecnologías inalámbricas en ambientes al aire libre. Revista Mutis, 8(2), 57-72. https://doi.org/10.21789/22561498.1404

Belmonte-Espejo., P. \& Miralles-Martínez, P. (2004). El impacto social y ambiental de las redes de telefonía móvil. Scripta Nova Revista Electrónica de Geografía y Ciencias Sociales, 8 .

Cáceres, V. (2016). La regulación ambiental: el caso de las antenas de telecomunicaciones en Argentina. Actualidad Jurídica Ambiental, 4-28.

CODIGO ORGÁNICO AMBIENTAL. Código Orgánico Ambiental Ley 0 Reg Oficial 983. (2018).

Cruz-Ornetta, V. (2017). La gestión ambiental de los residuos sólidos de la telefonía móvil. Paideia, 3(4), 60-69. https://doi.org/10.31381/paideia.v3i4.1015

D’Almeida, F., \& Margot, D. (2018). La evolución de las telecomunicaciones móviles en América Latina $\mathrm{y}$ el Caribe. BID Invest, (4), 54. Retrieved from https://www.idbinvest.org/sites/default/files/201809/tn4_spa_la_evolucion_de_las_telecomunicaciones_moviles_2018.pdf

Díaz-Martín, M. (2001). Los impactos de las estaciones de telefonía móvil. Informes de La Construcción, 53, 47-52. https://doi.org/10.3989/ic.2001.v53.i476.649.

Hyland, G. J. (2000). Physics and biology of mobile telephony. Lancet, 356(9244), 18331836. https://doi.org/10.1016/S0140-6736(00)03243-8

Ministerio de Ambiente. (2013). Estudio de potenciales impactos ambientales y vulnerabilidad. Retrieved from http://www.ambiente.gob.ec/wpcontent/uploads/downloads/2013/03/PART11.pdf

Piñeres-Espitia, G. D. (2010). Telefonía celular un vistazo a una radiobase en Colombia. Inge-Cuc, 6(1), 131-142.

Silva-Chuquimarca, C. T. (2017). Evaluación del impacto social y visual, de las estaciones de telefonía móvil en la ciudad de Quito, y propuesta para mejorar la gestión. Escuela politécnica nacional. 


\section{PARA CITAR EL ARTÍCULO INDEXADO.}

Llanos Campaña, D. (2019). Evaluación de impacto ambiental de una radio bases celulares instalada desde el nivel del suelo en el Ecuador. Ciencia Digital, 3(4.2), 179-190. https://doi.org/10.33262/cienciadigital.v3i4.2.1022

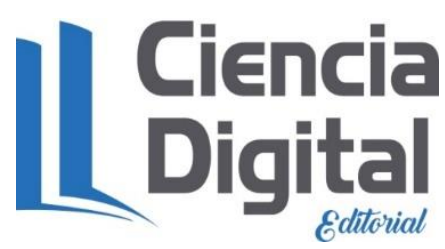

El artículo que se publica es de exclusiva responsabilidad de los autores y no necesariamente reflejan el pensamiento de la Revista Ciencia Digital.

El artículo queda en propiedad de la revista y, por tanto, su publicación parcial y/o total en otro medio tiene que ser autorizado por el director de la Revista Ciencia Digital.
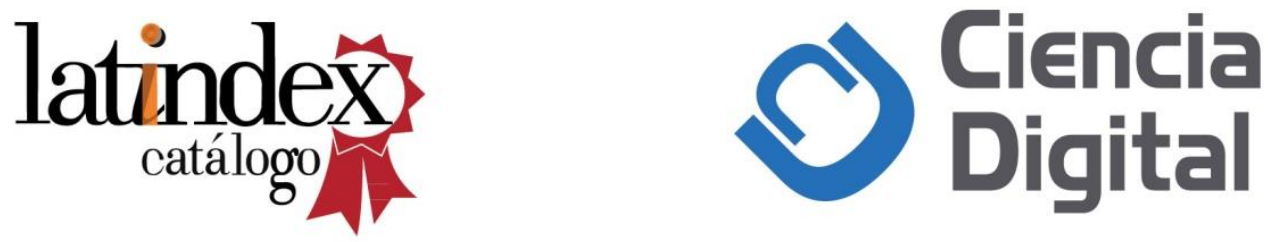